\title{
Chromatin-unstable boar spermatozoa have little chance of reaching oocytes in vivo
}

\author{
Florencia Ardón ${ }^{1}$, Dietmar Helms ${ }^{1}$, Evrim Sahin ${ }^{1}$, Heinrich Bollwein², Edda Töpfer-Petersen ${ }^{3}$ \\ and Dagmar Waberski ${ }^{1}$ \\ ${ }^{1}$ Unit for Reproductive Medicine of Clinics for Pigs and Small Ruminants, ${ }^{2}$ Clinic for Cattle and ${ }^{3}$ Institute for \\ Reproductive Biology, University of Veterinary Medicine Hannover, Bünteweg 15, D-30559 Hannover, Germany \\ Correspondence should be addressed to D Waberski; Email: dagmar.waberski@tiho-hannover.de
}

\begin{abstract}
In the present study, the prevalence of chromatin instability in the fertilizing-competent sperm population in the porcine oviduct in vivo was examined through qualitative analysis of the chromatin structure status of accessory boar sperm found in in vivo-derived embryos. The binding of chromatin-unstable sperm to oviductal epithelium in vitro was also studied. To examine the sperm chromatin state, a modified fluorescence microscopic sperm chromatin structure assay was used. Among a population of 173 fertile boars, individuals were selected for according to their chromatin status: 25 animals showed more than $\mathbf{5 \%}$ of chromatin-unstable sperm in their ejaculates, and 7 showed consistently elevated percentages of chromatin-unstable sperm in three successively collected semen samples. A positive correlation was found between incidence of chromatin instability and attached cytoplasmic droplets $(r=0.44, P<0.01)$. Analyses of accessory spermatozoa from in vivo-derived embryos demonstrated that the proportion of chromatin-unstable sperm was significantly $(\boldsymbol{P}<0.05)$ reduced in the population of fertilizing-competent sperm in the oviduct compared with the inseminated sperm. Populations of sperm bound to the oviduct in vitro had significantly $(\boldsymbol{P}<\mathbf{0 . 0 5})$ lower percentages of chromatin instability than in the original diluted semen sample. In conclusion, numbers of sperm with unstable chromatin are reduced in the oviductal sperm reservoir, possibly because of associated changes in the plasma membrane that prevent sperm from binding to the oviductal epithelium. We conclude that in vivo the likelihood that sperm with unstable chromatin will reach the egg and fertilize it is low.
\end{abstract}

Reproduction (2008) 135 461-470

\section{Introduction}

Of the millions of inseminated sperm, very few will interact with the oocyte and be able to penetrate the zona pellucida. This reduction is caused by strict mechanisms of sperm selection in the female reproductive tract, which ensure that only sperm with high fertilization potential will be able to reach and penetrate the oocyte (Hunter 1984, Hunter et al. 1991). Passage through the narrow folds of the uterotubal junction at estrus is achieved mainly by sperm with vigorous motility and regular shape. Recently, it was shown that the mammalian sperm reservoir in the caudal oviductal isthmus not only serves as a storage site for sperm from the moment of insemination until ovulation, but also plays a key role in the selection of sperm (Thomas et al. 1994, Lefebvre \& Suarez 1996, Mburu et al. 1996, Fazeli et al. 1999, Petrunkina et al. 2001a, 2001b, Gualtieri \& Talevi 2003). Some sperm with specific hidden defects, however, may appear to be normal with respect to the traits selected by the female reproductive tract, such as motility and morphology; therefore, it is possible for them to bypass the selection mechanism. Such sperm may be able to penetrate the zona pellucida, but have deficiencies that prevent either the fulfillment of fertilization or normal embryo development (Dejarnette et al. 1992, Thundathil et al. 2000). Loosely packed chromatin and damaged DNA is commonly regarded as an example of this. Normally, sperm chromatin structure is highly compact and stable, thus quite able to protect its genetic integrity during the transport of sperm through the male and female reproductive tracts (Ward \& Coffey 1991). It has also been suggested that stable chromatin structure provides sperm with a rigidity that enables them to penetrate the zona pellucida (Bedford 1998). Information from several species, including porcine, show no constant or only a poor-to-moderate correlation between chromatin abnormality and conventional semen quality tests (human: Evenson et al. 1991, boar: Hernández et al. 2006, bull: Ballachey et al. 1987, horse: Kenney et al. 1995). Most studies agree that chromatin instability in individual sperm is not closely associated with deficient motility or morphological aberration that would hinder such sperm from entering the oviduct. As a consequence, chromatin-unstable sperm with regular motility and morphology are believed 
to be able to reach and to interact with the oocyte in vivo. In the context of human-assisted reproduction techniques, the extent of chromatin and/or DNA defects in the semen samples has been linked to impairment of fertilization and embryo development as well as to reduced numbers of live offspring (see Larson-Cook et al. 2003 and references therein). Insemination trials in livestock indicate a relationship between the occurrence of sperm DNA damage and fertility (reviewed by Evenson \& Wixon 2006). However, the potential ability of sperm with damaged DNA to fertilize oocytes and the consequences for embryonic development have not been widely studied (Fatehi et al. 2006). Recently, it has been shown that bovine sperm with DNA strand breaks induced by X-ray irradiation allowed normal in vitro fertilization (IVF) to occur but led to a developmental block due to apoptosis after the second or third embryo cleavage (Fatehi et al. 2006). It is not known, however, whether and to what extent chromatinunstable sperm contributes to the fertilization process in vivo, where selective barriers of the female tract have to be overcome before the sperm meets the egg.

The objective of the present study was to investigate the prevalence of chromatin instability in the fertilizingcompetent sperm population in the porcine oviduct in vivo through qualitative analysis of the chromatin structure status of accessory sperm found in in vivo-derived embryos. In addition, we studied whether, and to what extent, boar sperm with unstable chromatin have the capacity to bind to the oviductal epithelia in vitro; similar binding in vivo is an essential step in establishing the oviductal sperm reservoir.

\section{Results}

\section{Reliability of mfSCSA data}

Correlation between the flow cytometric SCSA and the mfSCSA

The percentage of chromatin-unstable sperm in the modified fluorescence microscopic sperm chromatin structure assay (mfSCSA) was significantly positively correlated $(r=0.95, P<0.001)$ to the percentage of chromatin instability as evaluated by flow cytometric SCSA (Fig. 1). Values $(n=40)$ lay in a range between 1 and $12 \%$ chromatin instability.

\section{Slides evaluated on two different occasions}

The mean percentages of sperm with unstable chromatin found for each evaluation day were 11.1 and $12.5 \%$ for the first and second evaluations respectively. The mean difference between both evaluations was 1.37. The day of evaluation did not affect evaluation results $(P>0.05)$. The correlation between the evaluations was $r=0.97$ $(P<0.01)$.

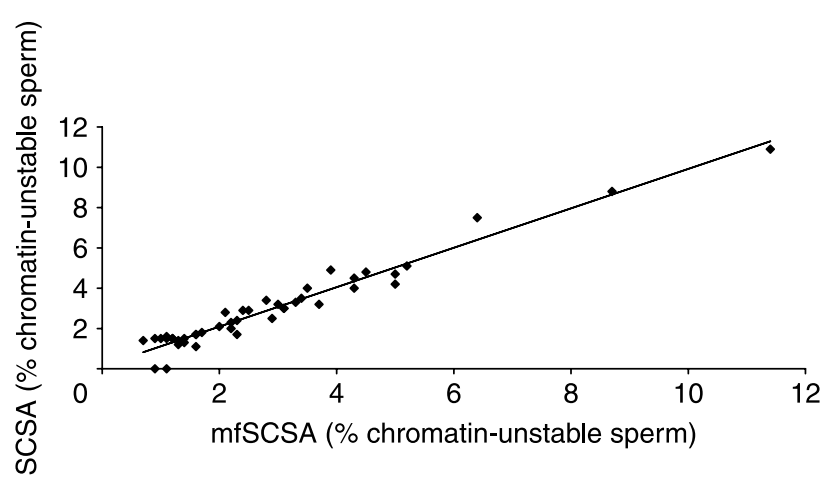

Figure 1 Correlation between the percentages of chromatin-unstable sperm detected by flow cytometric SCSA and those detected by the modified fluorescence microscopic SCSA (mfSCSA). Data are presented for 40 independent ejaculates; $r=0.95, P<0.001$.

\section{Presence of chromatin-unstable sperm in boar ejaculates}

\section{Prevalence of chromatin instability}

$85.5 \%$ of the semen samples evaluated $(n=173$, one per boar) showed $<5 \%$ of sperm with unstable chromatin. The range was $0.1-19 \%$ and the mean for all samples was $3.2 \%$ chromatin-unstable sperm.

\section{Persistency of chromatin instability}

In 15 boars showing $>5 \%$ chromatin-unstable sperm in an initial semen sample, two further ejaculates collected on successive weeks were investigated. Six of these boars showed elevated percentages of chromatinunstable sperm only in the first semen sample. However, seven showed constant elevated numbers of chromatinunstable sperm in all three samples. The mean percentage of chromatin instability in boars with three successive samples containing $>5 \%$ chromatinunstable sperm was significantly higher $(P<0.05)$ than in boars with either one or two samples with enhanced chromatin instability (Table 1).

\section{Association between chromatin instability and other sperm defects}

The percentages of attached cytoplasmic droplets and total abnormalities were significantly higher $(P<0.01)$ in ejaculates with high $(\geq 5 \%)$ percentages of sperm with unstable chromatin than in those showing low $(<5 \%)$ percentages of this defect (Table 2). The percentage of sperm with unstable chromatin correlated significantly with the percentage of sperm with cytoplasmic droplets ( $r=0.44, P<0.01, n=128$ ejaculates from 54 boars; Fig. 2). No correlation was found between the percentage of sperm with unstable chromatin and other standard sperm parameters, i.e., motility and morphological abnormalities other than attached cytoplasmic droplets (data not shown). 
Table 1 In 15 boars with $>5 \%$ of chromatin-unstable sperm in an initial diluted semen sample, the chromatin status in two further ejaculates collected in successive weeks was investigated. Data are based only on ejaculates with $>5 \%$ chromatin-unstable sperm.

\begin{tabular}{l}
$\begin{array}{l}\text { Presence of }>\mathbf{5} \% \text { chromatin-unstable sperm in } \\
\text { three semen samples collected in successive weeks }\end{array}$ \\
\hline 1 st semen sample only \\
1 st and 2 nd semen sample \\
1 st to 3 rd semen sample \\
${ }^{*,+}$ Significantly different $(P<0.05)$. \\
Chromatin stability in sperm bound to oviductal \\
epithelium in vitro
\end{tabular}

\section{Sperm parameters of boars 1-4}

Semen of two control boars with a low prevalence of chromatin instability (boars 1 and 2) and of two boars with enhanced percentages of chromatin instability in consecutive ejaculates (boars 3 and 4) were used. Sperm data are given in Table 3. Boars 3 and 4 had significantly higher $(P<0.05)$ percentages of sperm with cytoplasmic droplets compared with the control boars. Other morphological abnormalities and sperm motility did not differ between the boars.

Chromatin status after Percoll washing and after release of oviduct-bound sperm

In boars 3 and 4, Percoll gradient centrifugation of the semen samples significantly reduced $(P<0.05)$ the percentage of sperm with unstable chromatin. In all four boars, sperm bound to the oviductal pieces had significantly lower percentages $(P<0.05)$ of chromatin instability than the original semen samples or the Percoll-treated sperm (Fig. 3).

\section{Chromatin instability in accessory sperm from in vivo- derived embryos}

\section{Sperm parameters of boars 5-7}

Semen of two boars with $>5 \%$ chromatin-unstable sperm (boars 5 and 6) and of one control boar (boar 7) was used. Sperm data are given in Table 4 . Boars 5 and 6 had significantly higher $(P<0.05)$ percentages of sperm with cytoplasmic droplets compared with the control boar. Other morphological abnormalities and sperm motility did not differ between the boars.

\section{Chromatin status in accessory sperm from in vivo-} derived embryos

All gilts inseminated were fertilized. The overall recovery rate of oocytes and embryos was $82 \%$. Fertilization rate based on normal developed embryos varied between 74.4 and $100.0 \%$. A total of 159 embryos (24-54 per boar) and 19839 accessory spermatozoa (4036-8514 per boar) were analyzed. Results are given in Fig. 4. In the boars showing $>5 \%$ of sperm with unstable chromatin, the percentage of chromatin instability was lower $(P<0.05)$ in accessory sperm than in diluted semen used for insemination $(6.5 \%$ in diluted semen and $1.9 \%$ in accessory sperm in boar $5 ; 8.9 \%$ in diluted semen and $1.4 \%$ in accessory sperm in boar 6$)$. In the control boar (boar 7), no significant difference was observed $(2.1 \%$ in diluted sperm and $1.0 \%$ in accessory sperm). Boar individuals did not exert any effect on the percentage of chromatin instability in the accessory sperm, the percentages being similar for all boars. The quality of the embryo (i.e., normal versus degenerated) was not related to the percentage of chromatin-unstable accessory sperm (1.4\% for normal and $1.3 \%$ for degenerated embryos; Table 5).

\section{Discussion}

Male fertility in domestic animals can be defined as the capacity of their sperm to establish pregnancies with adequate numbers of vital, developing, competent embryos in a large number of females. Insemination of large numbers of sperm translates into a selection of a few highly fertile sperm that will be able to reach and interact with the oocytes. Current opinions about sperm assessment technologies emphasize the role of the complex natural sperm selection mechanisms along the barriers in the female tract. Semen quality assessment methods may be most informative when a degree of sperm selection is incorporated (Holt \& van Look 2004). Recently, chromatin instability in sperm has gained the research interest of spermatologists. A number of studies in various species indicate that chromatin instability is related to subfertility. To date, however, the mechanisms and the extent of this

Table 2 Standard sperm parameters in diluted ejaculates (means \pm s.D.) with less than or more than $5 \%$ chromatin-unstable sperm.

\begin{tabular}{lll}
\hline & \multicolumn{2}{c}{ Chromatin-unstable sperm } \\
\cline { 2 - 3 } & $<5 \%$ & $>5 \%$ \\
\hline Boars $(n)$ & 38 & 16 \\
Ejaculates $(n)$ & 77 & 51 \\
Chromatin-unstable sperm (\%) & $2.5 \pm 1.2^{*}$ & $10.2 \pm 5.0^{+}$ \\
Motility (\%) & $79.9 \pm 5.0^{*}$ & $80.1 \pm 6.5^{*}$ \\
Morphologically abnormal sperm & & \\
Cytoplasmic droplets (\%) & $12.3 \pm 10.4^{*}$ & $25.3 \pm 18.3^{+}$ \\
Other than cytoplasmic droplets (\%) & $7.3 \pm 8.2^{*}$ & $8.8 \pm 10.1^{*}$ \\
\hline
\end{tabular}

${ }^{*}{ }^{\dagger}$ Significantly different $(P<0.05)$. 


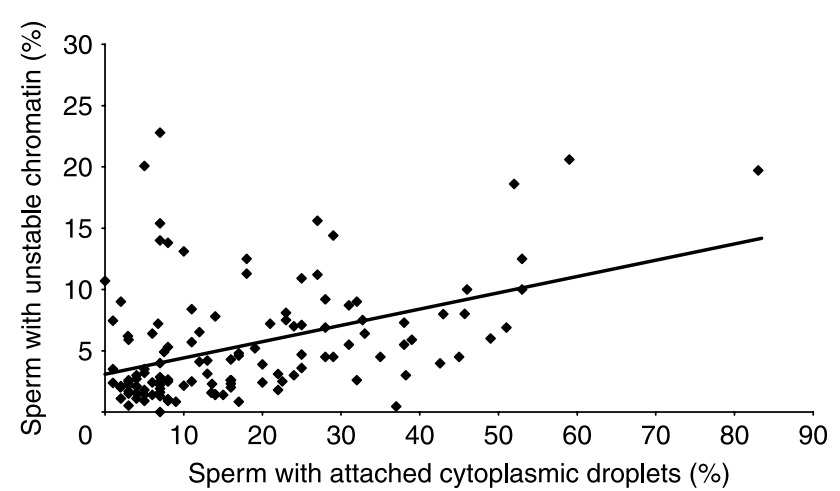

Figure 2 Correlation between the percentages of sperm with unstable chromatin and sperm with attached cytoplasmic droplets; $r=0.44$, $P<0.01, n=128$ ejaculates from 54 boars.

relationship are still unclear, particularly in boars, where only little information about the relevance of chromatin instability is available (Evenson et al. 1994). The main results of the present study demonstrate that the number of chromatin-unstable boar sperm is reduced in the population of fertilizing-competent sperm in the oviduct when compared with inseminated sperm. The in vivo model used in this study was based on the accessory sperm found in the zona pellucida of day 3-5 embryos. Accessory sperm are those found entrapped in the zona pellucida. The zona block to polyspermy in the pig is established only in the innermost portion of the zona pellucida, thus allowing other sperm to penetrate the outer zona as long as the embryo remains in the oviduct (Hunter 1997). Although accessory sperm are not directly involved in fertilization, they are believed to reflect the size of the functional sperm reservoir and the displacement of viable sperm from the oviductal sperm reservoir towards the site of fertilization (Hancock 1957, Hunter \& Wilmut 1984). Thus, they represent the highly selected population of fertilizingcompetent sperm in the oviduct; in pigs, their number is not affected by the embryo's subsequent quality (Ardón et al. 2005). Since the population of sperm obtained from the zona pellucida and the oviductal tissue was small, a modified fluorescence microscopic version of the SCSA (Evenson \& Jost 2000) was established. Termed mfSCSA, this was based on the microscopic acridine orange test (AOT; Tejada et al. 1984, Kosower et al. 1992, Acevedo et al. 2002), and included the detection of fluorescence by objective digital image analysis. The mfSCSA proved appropriate for the study of small populations of sperm and where sperm cannot be separated from other cells. In the present study, in boars with elevated amounts of chromatin-unstable sperm in their ejaculate, significantly lower percentages $(<3 \%)$ of those sperm were found in the accessory sperm population. Because the chromatin is enclosed within the sperm head, a direct communication between chromatin and the female tract is unlikely. The female reproductive tract is unlikely to be able to assess the sperm DNA quality directly, and thus selection is more probably based on sperm phenotype and function (Holt \& van Look 2004). Our study has found that sperm bound to and released from the oviducts in vitro have lower percentages of chromatin instability than those in the added semen sample. This concurs with the findings of Ellington et al. (1999) that cryopreserved human sperm attached to cultured homologous oviduct epithelia cells in vitro had fewer abnormalities in chromatin structure compared with sperm that were not attached. Together, these results suggest that chromatin instability may be associated with plasma membrane characteristics that hinder the binding of such sperm to the oviduct. A lower capacity to bind to the oviduct would lower the number of chromatinunstable sperm found in the reservoir, which would likely reduce the chances of this class of sperm to interact with the oocytes. An additional explanation for the reduced number of chromatin-unstable sperm in the vicinity of the egg as seen in our in vivo study would be reduced entry of such sperm in the oviduct due to impaired passage of the physicomechanical barriers of the uterotubal junction. Support for this suggestion is given by the observation that human cervical mucus can act in vitro as a selective barrier against human spermatozoa carrying fragmented DNA and chromatin structural abnormalities (Bianchi et al. 2004). However, the degree of this selection is likely to depend on the association between chromatin instability and other malformations of the sperm. It remains doubtful whether chromatin-unstable, but otherwise normal, sperm are selected at the uterotubal junction in vivo. Sperm used for IVF and intracytoplasmic sperm injection (ICSI) will

Table 3 Chromatin status and standard sperm parameters of diluted semen of boars 1-4 (means \pm s.D.) used for co-incubation with oviductal strips in vitro to generate oviduct-bound sperm for further analysis of the chromatin status. Boars 1 and 2 were considered as control boars.

\begin{tabular}{|c|c|c|c|c|}
\hline & \multicolumn{4}{|c|}{ Boar no. } \\
\hline & 1 & 2 & 3 & 4 \\
\hline Ejaculates $(n)$ & 4 & 4 & 3 & 3 \\
\hline Chromatin-unstable sperm (\%) & $1.6 \pm 0.5^{*}$ & $2.4 \pm 0.6^{*}$ & $15.4 \pm 1.0^{+}$ & $8.4 \pm 2.4^{\ddagger}$ \\
\hline Motility $(\%)$ & $81.3 \pm 6.3^{*}$ & $81.3 \pm 2.5^{*}$ & $76.7 \pm 5.8^{*}$ & $81.7 \pm 2.9^{*}$ \\
\hline \multicolumn{5}{|l|}{ Morphologically abnormal sperm } \\
\hline Cytoplasmic droplets (\%) & $7.8 \pm 5.0^{*}$ & $5.3 \pm 3.3 *$ & $26.7 \pm 1.5^{+}$ & $31.7 \pm 5.7^{+}$ \\
\hline Other than cytoplasmic droplets (\%) & $3.5 \pm 4.4^{*}$ & $2.8 \pm 2.2^{*}$ & $3.3 \pm \overline{2} .5^{*}$ & $1.0 \pm 1.7^{*}$ \\
\hline
\end{tabular}

${ }^{*,+, \neq}$ Significantly different $(P<0.05)$. 


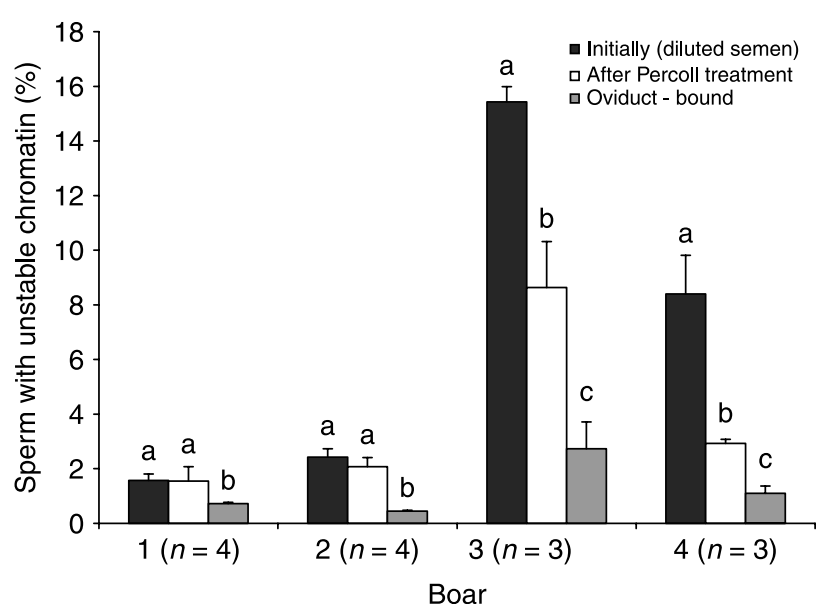

Figure 3 Percentages of sperm with unstable chromatin structure in diluted semen, after Percoll treatment and after oviduct binding. Bars represent S.E.M. ${ }^{\mathrm{a}-\mathrm{c}}$ Significantly different $(P<0.05)$ within a boar.

not encounter the selective barriers in the uterus and the oviduct, so that there is a higher chance that chromatinunstable sperm will be used to fertilize the oocytes. The defective chromatin structure does not seem to impair the ability of spermatozoa to fertilize, but evidence has been provided that such defects cause early embryonic death, already affecting the first cleavage divisions (Larson-Cook et al. 2003, Fatehi et al. 2006). The prevalence as well as the incidence of abnormal chromatin structure in spermatozoa from human and domestic animal species varies greatly. In the present and in other studies (López-Fernández et al. 2008), the incidence of chromatin instability in boar sperm has been found to be generally low and has not shown a constant correlation with any one sperm defect except for attached cytoplasmic droplets. A correlation between the susceptibility of DNA to denaturation and sperm cytoplasmic droplets was also observed in human (Fischer et al. 2003). In our study, all four experimental boars with consistently elevated percentages of chromatin-unstable sperm also revealed enhanced percentages

Table 4 Chromatin status and standard sperm parameters of diluted semen of boars 5-7 (means \pm s.D.) used for insemination to generate accessory spermatozoa from in vivo-derived embryos for further analysis of the chromatin status. Boar 7 was considered as control boar.

\begin{tabular}{llll}
\hline & \multicolumn{3}{c}{ Boar no. } \\
\cline { 2 - 4 } & 5 & 6 & 7 \\
\hline Ejaculates $(n)$ & 4 & 6 & 4 \\
$\begin{array}{l}\text { Chromatin-unstable } \\
\quad \text { sperm (\%) }\end{array}$ & $6.5 \pm 2.4^{*}$ & $8.9 \pm 8.8^{*}$ & $2.1 \pm 1.1^{+}$ \\
Motility (\%) & $85.0 \pm 0.0^{*}$ & $82.0 \pm 2.7^{*}$ & $78.8 \pm 8.5^{*}$ \\
$\begin{array}{l}\text { Morphologically abnormal sperm } \\
\quad \text { Cytoplasmic droplets (\%) }\end{array}$ & $28.0 \pm 4.6^{*}$ & $29.8 \pm 2.1^{*}$ & $4.3 \pm 2.5^{+}$ \\
$\quad \begin{array}{lll}\text { Other than cytoplasmic } \\
\quad \text { droplets (\%) }\end{array}$ & $8.0 \pm 2.6^{*}$ & $3.5 \pm 1.0^{*}$ & $4.5 \pm 3.3^{*}$ \\
\hline
\end{tabular}

\footnotetext{
${ }^{*}{ }^{+}$Significantly different $(P<0.05)$.
}

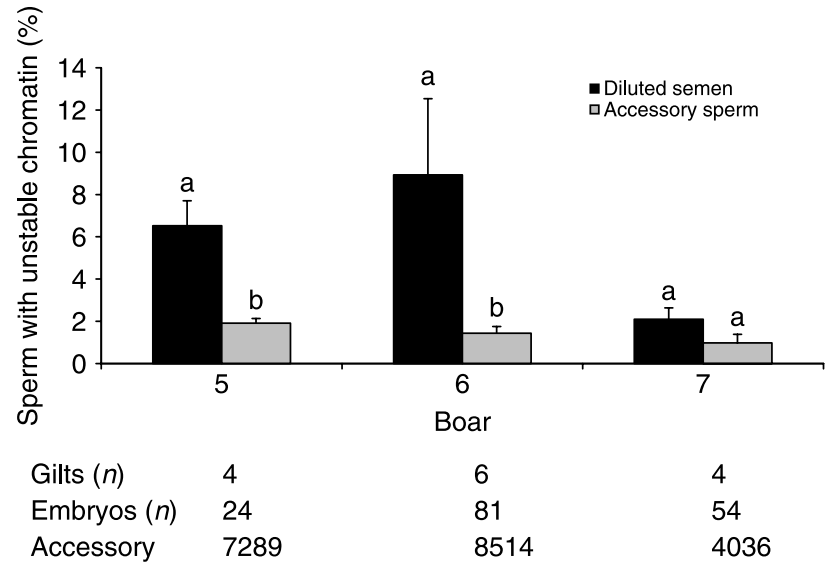

$\operatorname{sperm}(n)$

Figure 4 Percentages of sperm with unstable chromatin structure in diluted semen used for insemination and in the population of accessory sperm in resultant in vivo-derived embryos. Bars represent S.E.M.

${ }^{a, b}$ Significantly different $(P<0.05)$ within a boar.

of sperm with attached cytoplasmic droplets. Interestingly, as with chromatin-unstable sperm, binding to oviductal explants in vitro is negatively correlated with the percentage of sperm with plasma droplets (Petrunkina et al. 2001a, Waberski et al. 2006). The existence and the nature of the functional relationship between these observations remain unclear. During transit through the epididymis sperm chromatin stability is achieved by disulfide-mediated stabilization of protamines. It has been suggested that spermatozoa with red acridine orange fluorescence represent immature sperm (Kosower et al. 1992). Chromatin instability as detected with the SCSA may only show the 'tip of the iceberg' of a population of immature spermatozoa (Evenson et al. 2002). The presence of attached cytoplasmic droplets is indicative of sperm immaturity, and in some (at least) of these sperm the chromatin structure may not have reached its completely stabilized protamine configuration (Ward \& Coffey 1991, Kosower et al. 1992). Immaturity of sperm, therefore, may be associated with incomplete function of the sperm plasma membrane, which impairs binding to the oviductal epithelium, e.g., by a failure of interaction between protein/carbohydrate-binding sites on the sperm surface and glycosylated ligands on the oviductal epithelium.

Table 5 Chromatin status in accessory sperm (means \pm s.D.) from in vivo-derived normal and degenerated embryos.

\begin{tabular}{lll}
\hline & \multicolumn{2}{c}{ Type of embryo } \\
\cline { 2 - 3 } & Normal & Degenerated \\
\hline Embryos $(n)$ & 115 & 44 \\
Accessory sperm with & $1.4 \pm 0.7$ & $1.3 \pm 0.8$ \\
$\quad$ unstable chromatin $(\%)$ & & \\
\hline
\end{tabular}


However, it should be noted that the correlation between attached cytoplasmic droplets and chromatin-unstable sperm is significant but not very high $(r=0.502$, López-Fernández et al. 2008; $r=0.44$ in the present study), and the association between chromatin instability and the presence of cytoplasmic droplets in individual sperm has not been shown. Nevertheless, both defects could be indicative of deficiencies in plasma membrane function, resulting in a diminished ability of sperm to form the female sperm reservoir by binding to the oviduct. An association between maturational defects in plasma membrane function and sperm binding to oviductal epithelium has been indicated recently in bulls (Khalil et al. 2006). Whereas cytoplasmic droplets present a quite common sperm abnormality in boar ejaculates, the incidence of chromatin instability seems to be relatively low in commercial boar semen (López-Fernández et al. 2008). However, it has been suggested that even low numbers of sperm (5-10\%) with clearly disturbed chromatin structure may imply the presence of undiagnosed chromatin disturbances in a larger population of sperm (Evenson et al. 2002). Nevertheless, in the present study only 7 out of 173 tested boars showed more than $5 \%$ chromatin-unstable sperm consistently in three semen samples collected in successive weeks; eight further boars showed elevated numbers of chromatin-unstable sperm only occasionally in single or two successive ejaculates. This indicates that in pigs, different from species that are less selected for fertility, enhanced incidence of chromatin instability does not necessarily signal a chronic problem in the donor boar.

In conclusion, the results of this study suggest that numbers of boar sperm with unstable chromatin are reduced in the oviductal sperm reservoir in vivo, possibly because of associated changes in the plasma membrane, which prevent sperm from binding to the oviductal epithelium. Association with other sperm defects might also hinder the entrance of chromatinunstable sperm into the oviducts. Thus, in vivo the likelihood that boar sperm with unstable chromatin will reach the eggs and fertilize them is low.

\section{Materials and Methods}

\section{Chemicals}

Unless otherwise stated, chemicals were obtained from Merck and Carl Roth (Karlsruhe, Germany), were of high purity, and all solutions were prepared using double distilled water.

\section{Semen and oviducts}

The semen was generously provided by a local artificial insemination (Al) center and delivered overnight using an express delivery service. The semen doses had been diluted at the AI center in Beltsville Thawing Solution (Johnson et al. 1988) and contained a total of $2 \times 10^{9}$ sperm cells. The slaughterhouse of the city of Hannover donated the oviducts of multiparous sows that had been sent for commercial slaughter. Only oviducts from clinically normal genital tracts were collected.

\section{Animals}

The 14 gilts for the in vivo experiment were bought from a nearby commercial farm. They were 6-8 months old, weighted at least $90 \mathrm{~kg}$, and were housed in groups of two to three at the facilities of the Unit for Reproductive Medicine. The animals were treated according to the Animal Welfare Act of the German Federal Ministry, consistent with the International Guiding Principles for Biomedical Research Involving Animals as promulgated by the Society for the Study of Reproduction.

\section{Modified fluorescence microscopic sperm chromatin structure assay ( $\mathrm{mfSCSA}$ )}

Essentially, the methods of Evenson et al. (1980), Tejada et al. (1984), Kosower et al. (1992), and Acevedo et al. (2002) were combined while including essential modifications of our own.

\section{Media}

The citrate buffer solution was composed of $98.6 \mathrm{mM}$ trisodium citrate and $11.5 \mathrm{mM}$ EDTA $(\mathrm{pH}$ 6.8). The dithiothreitol/dimethyl sulfoxide (DTT/DMSO) solution was prepared by adding $3.2 \mathrm{ml}$ hydrated DMSO to $30 \mathrm{ml}$ trisodium citrate buffer solution containing $10 \mathrm{mM}$ DTT; the final DTT concentration was $8.6 \mathrm{mM}$. The Carnoy solution (Tejada et al. 1984) was prepared with methanol and acetic acid at a ratio of $2: 1$. The acridine orange staining solution was prepared by mixing $2.5 \mathrm{ml}$ of $376.2 \mathrm{mM}$ sodium phosphate dibasic stock solution, $40 \mathrm{ml}$ of $100 \mathrm{mM}$ citric acid monohydrate stock solution, and $10 \mathrm{ml}$ of $3.3 \mathrm{mM}$ acridine orange stock solution; all stock solutions were prepared in distilled water, kept at $4{ }^{\circ} \mathrm{C}$, and used within a month.

\section{Washing of semen and preparation of slides}

Four milliliters of semen were mixed with $2 \mathrm{ml}$ citrate buffer solution and centrifuged at $2100 \mathrm{~g}$ for $10 \mathrm{~min}$. The supernatant was discarded and $2 \mathrm{ml}$ citrate buffer added. The samples were mixed thoroughly and centrifuged once again at $2100 \mathrm{~g}$ for $10 \mathrm{~min}$. The supernatant was removed leaving a small amount for resuspension of the sperm pellet. A $10 \mu \mathrm{l}$ droplet of resuspended sperm was placed on a Superfrost Plus slide (Carl Roth) and smeared using another slide. The slides were air dried for at least $20 \mathrm{~min}$ and kept refrigerated until further processing.

\section{Disulfide reduction (chromatin decondensation)}

This step took place under an extractor at a temperature between 18 and $23^{\circ} \mathrm{C}$. The slides were placed horizontally on test tube racks, taking care that they were as level as possible. Then, each slide was completely covered with $2 \mathrm{ml}$ DTT/DMSO solution. They were left to react for $30 \mathrm{~min}$. Then, each slide was rinsed with citrate buffer using a wash bottle and placed in a Hellendahl vertical glass stain jar, previously filled with $30 \mathrm{ml}$ 
buffer. The slides were left in the jar for $10 \mathrm{~min}$, then taken out, wiped once on the sides and back with absorbent paper, and placed vertically against the test tube racks to air dry for $20 \mathrm{~min}$.

\section{Acid denaturation}

This step took place at room temperature under an extractor while preventing direct contact of the samples with light. Sixty milliliters of Carnoy solution were prepared in a vertical Hellendahl glass stain jar while the slides were being air dried. The jar was covered with an aluminum foil. The air-dried slides were placed in the jar and left to react for $100 \mathrm{~min}$; then they were taken out, wiped once on the sides and back with absorbent paper, and placed vertically against the test tube racks to air dry for at least $10 \mathrm{~min}$.

\section{Staining with acridine orange}

The slides were protected from direct contact with light during this step, which was carried out strictly at $4{ }^{\circ} \mathrm{C}$. A pre-cooled, vertical Hellendahl glass stain jar was placed in a pre-cooled water bath. The acridine orange staining solution was prepared in the stain jar. The slides were placed in the jar and left for 20 min. Then slides were taken out, wiped once on the sides and back with absorbent paper, and placed in a pre-cooled jar filled with $30 \mathrm{ml}$ citrate buffer. They were left in the buffer solution for $10 \mathrm{~min}$. Then they were taken out, wiped again, and placed vertically against the test tube racks to air dry for at least $20 \mathrm{~min}$. Once the slides were dry, they were stored in a slide storage box at $4{ }^{\circ} \mathrm{C}$ until evaluation.

An aliquot of a frozen control sample of sperm was processed with each staining batch to assure that the factors related to each work day, such as room temperature and humidity, would not influence the results. Temperature, humidity, and any abnormal circumstances were noted to determine their influence on the slides' quality.

\section{Fluorescence microscopy and digital image analysis}

The Zeiss Axioscope fluorescence microscope (Jena, Germany) was set to 200×, phase 2, 450-490 filter, FT 510, LP 520. The AnalySIS 3.0 (Soft Imaging System, Münster, Germany) computer software was used for the evaluation. The software was set to recognize as sperm particles bigger than 700 pixels, to prevent the detection of small dust particles as sperm.

The classification of sperm is based on the fluorescence acquired by the DNA after acridine orange staining. Acridine orange intercalates between the stacked bases of double-stranded (unfragmented, stable) DNA (ds-DNA) and fluoresces green at $530 \mathrm{~nm}$. In the case of single-stranded (fragmented) DNA (ss-DNA), stacked arrays of acridine orange bind to the phosphate backbone of the nucleotide and fluoresces red at $640 \mathrm{~nm}$.

The exposure time for the digital camera Olympus DP 50 (Olympus, Hamburg, Germany) was set at $\sim 40 \mathrm{~ms}$, the light sensitivity to ISO 2000, and the correction of the color scale was set to $0.9,0.9$, and 1.57 for red, green, and blue respectively. The fields were selected for evaluation using the 'live-window' feature of the software and photographed at a resolution of $2776 \times 2074$ pixels.
Threshold values for each fluorescence channel were set for the classification of sperm. For this, representative red and green sperm in a field were selected and their intensity profile was evaluated. The threshold values referred to the quantity of green and red colors found in green- and red-appearing sperm. After the threshold values were chosen, the sperm were classified either automatically or manually as either chromatin stable (ds-DNA, green fluorescence) or chromatin unstable (ss-DNA, red fluorescence). The same threshold values were used for all pictures taken from a given slide. Manual classification was used where few sperm per field were available or unspecific fluorescence was present from oviductal or embryonic cells. A minimum of four fields and at least 500 sperm cells per slide were analyzed. Regions with unequal staining, i.e., the edges and the center of the slide were not considered for evaluation.

\section{Reliability mfSCSA data}

The original microscopic AOT described by Tejada et al. (1984) was found to give variable results due to indistinct colors, rapid fading, and heterogenous slide staining (Evenson et al. 2002, Chohan et al. 2006). Considering the SCSA as gold standard (Chohan et al. 2006), two experiments were performed to show that the mfSCSA, as used in the present study, gave results comparable with the SCSA data. In the first experiment, the results of mfSCSA were compared with the results obtained from flow cytometric SCSA using split semen samples of 40 boar ejaculates snap frozen in liquid nitrogen. The ability to freeze raw or extended semen in liquid nitrogen without affecting SCSA parameters has been shown previously (Evenson \& Jost 1994, Evenson et al. 2002) and was confirmed in our own experiments (data not shown). The flow cytometric SCSA was carried out following the procedure described by Evenson \& Jost (1994) using the equipment, chemicals, and data analysis software in same laboratory as described by Schmid et al. (2003). In the second experiment, 30 slides obtained from the semen samples from 30 different boars were processed with the mfSCSA and each slide was evaluated on two different days. The first evaluation was performed at the day of processing and the second evaluation between 6 and 11 months later. During the time between evaluations, the slides had been kept in the dark at $4{ }^{\circ} \mathrm{C}$. Classification of green (chromatin-unstable) and red (chromatin-unstable) sperm were compared between the first and second days of analysis.

\section{Presence of chromatin-unstable sperm in boar ejaculates}

For the selection of boars with different percentages of chromatin-unstable sperm, single diluted semen samples from 173 boars were analyzed using the mfSCSA. In 54 boars, sperm morphology (as per Hancock 1956) and motility were additionally evaluated. In 15 boars with elevated percentages of chromatin-unstable sperm in the first semen sample, a further two ejaculates were collected and investigated in successive weeks. 


\section{Chromatin status in sperm bound to oviductal epithelium in vitro}

\section{Oviduct preparation}

Fallopian tubes were obtained at the local abattoir from sows at different cycle stages. The oviducts were transported to the laboratory in ice-cold PBS. The protocol essentially follows the oviductal explant assay as described by Petrunkina et al. (2001a) with few modifications. Briefly, oviducts were placed in a Petri dish containing ice-cold PBS, stripped from all surrounding tissue, and then opened longitudinally. Oviducts were then fixed with needles on a Petri dish filled with paraffin wax. To maximize the number of sperm bound to oviductal epithelium, long pieces (7-9 cm long, $0.6-0.8 \mathrm{~cm}$ wide) of isthmic longitudinal folds of the oviductal epithelia were dissected out under a stereomicroscope. For each assay, two such oviductal strips, each from a different sow, were then put in a small Petri dish containing $2.5 \mathrm{ml}$ modified TALP medium (Petrunkina et al. 2001a). The oviductal strips were refrigerated while the sperm were prepared.

\section{Sperm preparation}

This experiment compared semen from two boars with $<5 \%$ chromatin-unstable sperm (boar 1: $n=4$ ejaculates and boar 2 : $n=4$ ejaculates) with semen from two further boars with $>5 \%$ chromatin-unstable sperm in all ejaculates (boar 3: $n=3$ ejaculates and boar 4: $n=3$ ejaculates). Sperm samples were evaluated for motility and morphology using the criteria of Krause (1965). Sperm data are given in Table 3. Ten milliliters of diluted semen containing $25 \times 10^{6} \mathrm{sperm} / \mathrm{ml}$ were subjected to centrifugation at $170 \mathrm{~g}$ for $10 \mathrm{~min}$. Four milliliters of the resuspended sperm pellet were subjected to discontinuous (70/35) Percoll gradient centrifugation (Harrison et al. 1993). The sperm pellet was resuspended with $500 \mu \mathrm{l}$ modified Androhep without EDTA (Petrunkina et al. 2001a) and placed in a $1.5 \mathrm{ml}$ Eppendorf vial.

\section{Co-incubation of sperm and oviductal strips}

The Eppendorf vial with resuspended sperm and the Petri dish containing the pair of oviductal strips in $3.5 \mathrm{ml} \mathrm{TALP}$ medium were equilibrated at $39^{\circ} \mathrm{C}$ for $5 \mathrm{~min}$ in a humidified atmosphere containing $5 \% \mathrm{CO}_{2}$. Five hundred microliters of resuspended semen with a concentration of $35 \times 10^{7} \mathrm{sperm} / \mathrm{ml}$ were added to the Petri dish containing the oviductal strips. The samples were co-incubated for $30 \mathrm{~min}$ at $39^{\circ} \mathrm{C}$ in a humidified atmosphere containing $5 \% \mathrm{CO}_{2}$ to allow maximum sperm binding (Waberski et al. 2006). At the end of co-incubation, free-swimming spermatozoa were removed by extensive washings with TALP. The washings consisted of 'grasping' the strips with medium dissecting forceps, moving the strips vigorously around the Petri dish, and transferring them in another Petri dish containing $4 \mathrm{ml}$ TALP. Three washes in all were performed. The oviductal strips with the bound spermatozoa were then transferred to $1 \mathrm{ml} \mathrm{TALP}$ in a $1.5 \mathrm{ml}$ Eppendorf vial.

\section{Sperm release}

Virtually all sperm were released from oviductal strips by snap freezing in liquid nitrogen for $10 \mathrm{~min}$. After thawing at $39{ }^{\circ} \mathrm{C}$ for $5 \mathrm{~min}$, the oviductal strips were carefully removed. A few oviductal cells left in the suspension of released sperm were largely removed by centrifugation at $170 \mathrm{~g}$ for $10 \mathrm{~min}$. The released sperm (suspension in TALP) were submitted to the mfSCSA as described above.

\section{Chromatin status in accessory sperm from in vivo- derived embryos}

\section{Semen}

Semen from two boars with $>5 \%$ chromatin-unstable sperm (boars 5 and 6) and one boar with $<5 \%$ chromatin-unstable sperm (boar 7) in at least three successive ejaculates was used. Sperm samples were evaluated for motility and morphology using the criteria of Krause (1965). Aliquots of semen were snap frozen in liquid nitrogen for the mfSCSA. Sperm data are given in Table 4.

Estrus detection, insemination, and recovery of embryos/oocytes

This experiment was performed following the Hannover Gilt Model (HGM) guidelines (Ardón et al. 2003) with few modifications. The HGM includes the use of spontaneous ovulating gilts in their second estrus after stalling, estrus detection, and only one insemination in the presence of boars, and detection of ovulation via transabdominal ultrasound observation of the ovaries every $12 \mathrm{~h}$. In the present study, gilts were inseminated repeatedly with the purpose of maximizing the number of sperm recovered. The first insemination took place 12-18 $\mathrm{h}$ after first detection of estrus. Inseminations were repeated every $12 \mathrm{~h}$, as long as the gilt showed the standing reflex. Reproductive tracts of the gilts were recovered after slaughter, 3-5 days after ovulation. The reproductive tracts were kept at $37^{\circ} \mathrm{C}$ and immediately transported to the laboratory. The embryos and oocytes were flushed from the reproductive tracts and their morphology was evaluated as described by Ardón et al. (2003). As seen in earlier studies (Ardón et al. 2003, 2005), no sperm bound to the outer zona pellucida were present.

\section{Evaluation of the chromatin structure in accessory sperm}

The sperm chromatin structure was evaluated in the accessory sperm present in the zona pellucida of embryos. Accessory sperm are those found trapped in the zona pellucida; they interacted and partially penetrated the zona pellucida due to incomplete zona block in the pig (Hunter 1997). Embryos were mounted on Superfrost Plus slides using a micropipette. Slides were air dried and then refrigerated until treated. During this procedure, the zona pellucida bursts and the sperm disperse on the slide. The sperm on the slides were subjected to the mfSCSA as described above. The semen used for insemination, which had been snap frozen in liquid nitrogen, was subjected to the mfSCSA at the same time as the accessory sperm. 


\section{Statistical analysis}

Data are presented as arithmetic means with standard deviations calculated using the Excel software. All other statistical analyses were performed using SAS software (SAS Institute Inc., Cary, NC, USA). The correlation of two evaluations of the same slide was analyzed with the Spearman correlation (procedure CORR); the similarity between both evaluations was analyzed using the Kruskal-Wallis test (procedure NPAR1WAY) and the Student's $t$-test for paired samples. The following analyses were performed using the Wilcoxon test: effects of boars on sperm parameters, effects of sperm preparations (diluted, Percoll washed, oviduct bound, and accessory sperm) on the percentage of chromatin instability, effect of the embryo morphology on chromatin instability in accessory sperm. The Spearman correlation was also used to evaluate the correlation between chromatin instability and standard sperm parameters. The Spearman correlation and the Wilcoxon test were chosen because the data were not normally distributed. The criterion for significance was $P<0.05$.

\section{Acknowledgements}

Dedicated to our honored colleague Prof. Burkhard Meinecke. The authors are grateful to Dr R A P Harrison for critical reading of the manuscript and English language revision. This study was supported by the Development Association for Biotechnology Research (FBF), Germany. FA was supported by Consejo Nacional de Ciencia y Tecnología (CONACYT), Mexico, and German Academic Exchange Service (DAAD), Germany. The authors declare that there is no conflict of interest that would prejudice the impartiality of this scientific work.

\section{References}

Acevedo N, Bame J, Kuehn LA, Hohenboken WD, Evenson DP \& Saacke RG 2002 Sperm chromatin structure assay (SCSA) and sperm morphology. Proceedings of the 19th Technical Conference on Artificial Insemination \& Reproduction pp 84-90.

Ardón F, Döhring A, Le Thi X, Waberski D \& Weitze KF 2003 Assessing in vivo fertilizing capacity of liquid-preserved boar semen according to the Hanover Gilt Model. Reproduction in Domestic Animals 38 161-165.

Ardón F, Evert M, Beyerbach M, Weitze KF \& Waberski D 2005 Accessory sperm: a biomonitor of boar sperm fertilization capacity. Theriogenology 63 1891-1901.

Ballachey BE, Hohenbroken WD \& Evenson DP 1987 Heterogeneity of sperm nuclear chromatin structure and its relationship to bull fertility. Biology of Reproduction 36 915-925.

Bedford JM 1998 Mammalian fertilization misread? Sperm penetration of the eutherian zona pellucida is unlikely to be a lytic event Biology of Reproduction 59 1275-1287.

Bianchi PG, De Agostini A, Fournier J, Guidetti C, Tarozzi N, Bizzaro D \& Manicardi GC 2004 Human cervical mucus can act in vitro as a selective barrier against spermatozoa carrying fragmented DNA and chromatin structural abnormalities. Journal of Assisted Reproduction and Genetics 21 97-102.

Chohan KR, Griffin JT, Lafromboise M, De Jonge CJ \& Carrell DT 2006 Comparison of chromatin assays for DNA fragmentation evaluation in human sperm. Journal of Andrology 27 53-59.

DeJarnette JM, Saacke RG, Bame J \& Vogler CJ 1992 Accessory sperm: their importance to fertility and embryo quality, and attempts to alter their numbers in artificially inseminated cattle. Journal of Animal Science $\mathbf{7 0}$ 484-491.
Ellington JE, Evenson DP, Wright RW Jr, Jones AE, Schneider CS \& Hiss GA 1999 Higher quality human sperm in a sample selectively attach to oviduct (fallopian tube) epithelial cells in vitro. Fertility and Sterility $\mathbf{7 1}$ 924-929.

Evenson DP \& Jost LK 1994 Sperm chromatin structure assay: DNA denaturability. In Flow Cytometry Part B, 2 edn, Eds Z Darzynkiewicz, JP Robinson \& HA Crissman. Orlando, FL, USA: Academic Press.

Evenson D \& Jost L 2000 Sperm chromatin structure assay is useful for fertility assessment. Methods in Cell Science 22 169-189.

Evenson DP \& Wixon R 2006 Clinical aspects of sperm DNA fragmentation detection and male fertility. Theriogenology 65 979-991.

Evenson DP, Darzynkiewicz Z \& Melamed MR 1980 Relation of mammalian sperm chromatin heterogeneity to fertility. Science $\mathbf{2 1 0}$ $1131-1133$.

Evenson DP, Jost LK, Baer RK, Turner TW \& Schrader SM 1991 Individuality of DNA denaturation patterns in human sperm as measured by the sperm chromatin structure assay. Reproductive Toxicology 5 115-125.

Evenson DP, Thompson L \& Jost L 1994 Flow cytometric evaluation of boar semen by the sperm chromatin structure assay as related to cryopreservation and fertility. Theriogenology 41 637-651.

Evenson DP, Larson KL \& Jost LK 2002 Sperm chromatin structure assay: its clinical use for detecting sperm DNA fragmentation in male infertility and comparison with other techniques. Journal of Andrology 23 25-43.

Fatehi AN, Bevers MM, Schoevers E, Roelen BA, Colenbrander B \& Gadella BM 2006 DNA damage in bovine sperm does not block fertilization and early embryonic development but induces apoptosis after the first cleavage. Journal of Andrology 27 176-188.

Fazeli A, Duncan AE, Watson PF \& Holt WV 1999 Sperm-oviduct interaction: induction of capacitation and preferential binding of uncapacitated spermatozoa to oviductal epithelial cells in porcine species. Biology of Reproduction 60 879-886.

Fischer MA, Willis J \& Zini A 2003 Human sperm DNA integrity: correlation with sperm cytoplasmic droplets. Urology 61 207-211.

Gualtieri R \& Talevi R 2003 Selection of highly fertilization-competent bovine spermatozoa through adhesion to the fallopian tube epithelium in vitro. Reproduction 125 251-258.

Hancock JL 1956 The morphology of boar spermatozoa. Journal. Royal Microscopical Society 76 84-97.

Hancock JL 1957 The fertility of natural and of artificial matings in pig. Proceedings. Society for the Study of Fertility 9 146-158.

Harrison RA, Mairet B \& Miller NG 1993 Flow cytometric studies of bicarbonate-mediated $\mathrm{Ca}^{2+}$ influx in boar sperm populations. Molecular Reproduction and Development 35 197-208.

Hernández M, Roca J, Ballester J, Vázquez JM, Martínez EA, Johannisson A, Saravia F \& Rodríguez-Martínez H 2006 Differences in SCSA outcome among boars with different sperm freezability. International Journal of Andrology 29 583-591.

Holt WV \& Van Look KJW 2004 Concepts in sperm heterogeneity, sperm selection and sperm competition as biological foundations for laboratory tests of semen quality. Reproduction 127 527-535.

Hunter RHF 1984 Pre-ovulatory arrest and peri-ovulatory redistribution of competent spermatozoa in the isthmus of the pig oviduct. Journal of Reproduction and Fertility 72 203-211.

Hunter RHF 1997 Sperm dynamics in the female genital tract: interactions with the fallopian tube microenvironment. In Microscopy of Reproduction and Development: A Dynamic Approach, pp 35-45. Ed. PM Motta. Rome: Antonio Delfino Editore.

Hunter RHF \& Wilmut I 1984 Sperm transport in the cow: peri-ovulatory redistribution of viable cells within the oviduct. Reproduction, Nutrition, Development 24 597-608.

Hunter RHF, Flechon B \& Flechon JE 1991 Distribution, morphology and epithelial interactions of bovine spermatozoa in the oviduct before and after ovulation: a scanning electron microscope study. Tissue and Cel/ 23 641-656.

Johnson LA, Aalbers JG \& Grooton HJG 1988 Artificial insemination of swine: fecundity of boar semen stored in Beltsville TS (BTS), modified Modena (MM) or MR-A and inseminated on one, three and four days after collection. Zuchthygiene 23 49-55.

Kenney RM, Evenson DP, Garcia MC \& Love CC 1995 Relationship between sperm chromatin structure, motility, and morphology of ejaculated sperm and seasonal pregnancy rate. Biology of Reproduction Monograph Series 1 647-653. 
Khalil AAY, Petrunkina AM, Sahin E, Waberski D \& Töpfer-Petersen E 2006 Enhanced binding of sperm with superior volume regulation to oviductal epithelium. Journal of Andrology 27 754-765.

Kosower NS, Katayose H \& Yanagimachi R 1992 Thiol-disulfide status and acridine orange fluorescence of mammalian sperm nuclei. Journal of Andrology 13 342-348.

Krause D 1965 Contributions to the andrology of the bull. Deutsche Tierärzliche Wochenschrift 72 454-458.

Larson-Cook KL, Brannian JD, Hansen KA, Kasperson KM, Aamold ET \& Evenson DP 2003 Relationship between the outcomes of assisted reproductive techniques and sperm DNA fragmentation as measured by the sperm chromatin structure assay. Fertility and Sterility 80 895-902.

Lefebvre R \& Suarez SS 1996 Effect of capacitation on bull sperm binding to homologous oviductal epithelium. Biology of Reproduction 54 575-582.

López-Fernández C, Pérez-Llano B, García-Casado P, Sala R, Gosálbez A, Arroyo F, Fernández JL \& Gosálvez J 2008 Sperm DNA fragmentation in a random sample of the Spanish boar livestock. Animal Reproduction Science 103 87-98.

Mburu JN, Einarsson S, Lundeheim N \& Rodriguez-Martinez H 1996 Distribution, number and membrane integrity of spermatozoa in the pig oviduct in relation to spontaneous ovulation. Animal Reproduction Science 45 109-121.

Petrunkina AM, Gehlhaar R, Drommer W, Waberski D \& Töpfer-Petersen E 2001a Selective sperm binding to pig oviductal epithelium in vitro. Reproduction 121 889-896.

Petrunkina AM, Friedrich J, Drommer W, Bicker G, Waberski D \& TöpferPetersen E $2001 b$ Kinetic characterization of the changes in protein tyrosine phosphorylation of membranes, cytosolic $\mathrm{Ca}^{2+}$ concentration and viability in boar sperm populations selected by binding to oviductal epithelial cells. Reproduction 122 469-480.
Schmid TE, Kamischke A, Bollwein H, Niechlag E \& Brinkworth MH 2003 Genetic damage in oligozoospermic patients detected by fluorescence in situ hybridization, inverse restriction site mutation assay, sperm chromatin structure assay and the Comet assay. Human Reproduction 18 1474-1480.

Tejada RI, Mitchell JC, Norman A, Marik JJ \& Friedman S 1984 A test for the practical evaluation of male fertility by acridine orange (AO) fluorescence. Fertility and Sterility 42 87-91.

Thomas PG, Ball BA, Miller PG, Brinsko SP \& Southwood L 1994 A subpopulation of morphologically normal, motile spermatozoa attach to equine oviductal epithelial cell monolayers. Biology of Reproduction $\mathbf{5 1}$ 303-309.

Thundathil J, Meyer R, Palasz AT, Barth AD \& Mapletoft RJ 2000 Effect of the knobbed acrosome defect in bovine sperm on IVF and embryo production. Theriogenology 54 921-934.

Waberski D, Magnus F, Ardón F, Petrunkina AM, Weitze KF \& TöpferPetersen E 2006 Binding of boar spermatozoa to oviductal epithelium in vitro in relation to sperm morphology and storage time. Reproduction $131311-318$.

Ward WS \& Coffey DS 1991 DNA packaging and organization in mammalian spermatozoa: comparison with somatic cells. Biology of Reproduction 44 569-574.

Received 19 July 2007

First decision 4 September 2007

Revised manuscript received 6 December 2007

Accepted 3 January 2008 